\title{
The human myometrium differentially expresses mTOR signalling components before and during pregnancy: evidence for regulation by progesterone
}

\author{
Helen A Foster ${ }^{1}$, Julie Davies ${ }^{1}$, Ryan C Pink ${ }^{2}$, Serife Turkcigdem ${ }^{1}$, Anastasia Goumenou ${ }^{1}$, \\ David R Carter ${ }^{2}$, Nigel J Saunders ${ }^{3}$, Peter Thomas ${ }^{4}$, and Emmanouil Karteris ${ }^{1,}{ }^{*}$ \\ ${ }^{1}$ Biosciences, Centre for Cell and Chromosome Biology, Brunel University, Uxbridge, UB8, 3PH, \\ UK \\ ${ }^{2}$ School of Life Sciences, Oxford Brookes University, UK \\ ${ }^{3}$ Centre for Systems and Synthetic Biology, Brunel University, Uxbridge, UB8 3PH \\ ${ }^{4}$ Marine Science Institute, University of Texas at Austin, Port Aransas, Texas, TX 78373, USA
}

\begin{abstract}
Emerging studies implicate the signalling of the mammalian target of rapamycin (mTOR) in a number of reproductive functions. To this date, there are no data regarding the expression of mTOR signalling components in the human myometrium during pregnancy. We hypothesized that mTOR-related genes might be differentially expressed in term or preterm labour as well as in labour or non-labour myometria during pregnancy. Using quantitative RT-PCR we demonstrate for first time that there is a significant downregulation of mTOR, DEPTOR, and Raptor in preterm labouring myometria when compared to non-pregnant tissues taken from the same area (lower segment). We used an immortalised myometrial cell line (ULTR) as an in vitro model to dissect further mTOR signalling. In ULTR cells DEPTOR and Rictor had a cytoplasmic distribution, whereas mTOR and Raptor were detected in the cytoplasm and the nucleus, indicative of mTORC1 shuttling. Treatment with inflammatory cytokines caused only minor changes in gene expression of these components, whereas progesterone caused significant down-regulation. We performed a non-biased gene expression analysis of ULTR cells using Nimblegen human gene expression microarray $(n=3)$, and selected genes were validated by quantitative RT-PCR in progesterone treated myometrial cells. Progesterone significantly down-regulated key components of the mTOR pathway. We conclude that the human myometrium differentially expresses mTOR signalling components and they can be regulated by progesterone.
\end{abstract}

\section{Keywords}

mTOR; myometrium; progesterone; preterm labour 


\section{Introduction}

A number of recent studies implicates mammalian target of rapamycin (mTOR) signalling in reproductive functions. mTOR is a highly conserved Ser/Thr protein kinase that can form two distinct complexes termed mTORC1 and mTORC2 which can exert diverse but separate biological effects. The mTORC1 complex is sensitive to rapamycin treatment and contains the following components: mTOR, Raptor, G $\beta$ L (mLST8), PRAS40 and DEPTOR (DEPDC6, DEP-containing protein 6). This complex is capable of regulating translational processes and cell proliferation by altering the phosphorylation levels of downstream molecules such as p70 ribosomal S6 kinase 1 (S6K) and the eukaryotic initiation factor 4EBP1 [1-2]. The mTORC2 complex appears to be insensitive to rapamycin and contains: mTOR, Rictor, mSIN1, Protor-1, G $\beta$ L and DEPTOR. The functions of mTORC2 have not been as extensively studied as those of mTORC1. mTORC2 functions as a regulator of ATP kinases as it can phosphorylate Akt, protein kinase $\mathrm{Ca}$ and serum-and glucocorticoidinduced protein kinase [3] and has been proposed to function upstream of Rho GTPases to regulate the actin cytoskeleton [4].

At the placental level, mTOR signalling is a key mechanism linking maternal nutrient and growth factor concentrations to amino acid transport [5-7]. In growth restricted placentas, mTORC1 activity appears to be compromised as depicted by changes in the phosphorylation levels of S6K [7]. In addition, mTOR mediates human trophoblast invasion through regulation of matrix-remodelling enzymes and is associated with serine phosphorylation of STAT3 [8]. Our laboratory has demonstrated that during the process of syncytialization of cytotrophoblast (BeWo) cells, the expression of mTOR and 4EBP was down-regulated compared to unstimulated cells at the mRNA level [9]. We have also provided evidence for behavioural (i.e. emotional) influences on mTOR signalling at placental level as there was a significant inverse correlation between mRNA levels of placental DEPTOR and selfreported levels of stress in pregnant subjects [10].

The human myometrium undergoes differentiation in terms of cell number and size in two distinct stages of pregnancy: hyperplasia and hypertrophy [12]. The early phase of smooth muscle cell (SMC) proliferation (hyperplasia) which precedes implantation then gives rise to an intermediate synthetic phase [12]. During gestation the myometrium undergoes mainly hypertrophy, a phase of increased production of extracellular matrix proteins and subsequent increase of SMC size. The exact mechanisms that cause this particular switch from one phenotype to the other have not been fully elucidated. Shynlova et al., have demonstrated in a rodent model that the synthetic phase coincides with changes in the expression of certain caspases, as well as PARP [12].

mTOR has been implicated in the reconditioning of the myometrium during early stages of gestation in rodents. Jaffer et al., have provided conclusive evidence using an in vivo rat model that estradiol (E2) can induce the PI3K/mTOR signalling pathway in the myometrium, leading to uterine smooth muscle proliferation [11]. This study implicates mTOR signalling in events leading to the induction of myometrial hyperplasia during early gestation in a rodent model.

In contrast, to the best of our knowledge there are no reports on the expression of key mTOR signalling components in the human myometrium and in pathologies such as preterm labour. Preterm labour is defined as labour that begins before 37 completed weeks of pregnancy and is still the leading cause of perinatal morbidity and mortality in the developed world [13]. Despite advances in the diagnosis and molecular basis of this disease, efforts to prevent preterm birth have been compromised by a poor understanding of the underlying pathophysiology and not fully effective therapeutic interventions. However, we do know 
that preterm labour is associated with an inflammatory response and subsequently higher circulating levels of cytokines such as IL-1 $\beta$, IL-6, IL-8 and TNFa *14+. Interestingly, progesterone (P4) can exert anti-inflammatory effects in the context of intrauterine infection and also modulates myometrial contractile tone [15]. Previous studies have shown that the P4 plays a major anti-inflammatory role in human myometrial cells by antagonism of NF$\kappa \mathrm{B}$ activation of COX2 expression [16]. More recently, we have also shown that P4 inhibits the effects of IL-1 $\beta$ in two placental cytotrophoblast cell lines $* 17+$. We hypothesized that myometrial mTOR signalling might be altered in different stages (term or preterm) and contractile status (labour or non-labour) during pregnancy. We investigated this hypothesis by mapping the expression of mTOR, Rictor, Raptor and DEPTOR in clinical samples and assessing the effects of inflammatory cytokines and progesterone on the expression of these components as well as the activity of mTORC1 and mTORC 2 complexes.

\section{Materials and Methods}

\section{Subjects}

The study population consisted of pregnant women attending the Department of Obstetrics and Gynaecology, University Hospital, University of Crete. All participants gave informed consent to participate in the study and ethical approval was granted by the local ethics committee of the hospital as well as Brunel University. Women who were delivered by elective caesarean section at term and showed no signs of labour (uterine contractions and/ or cervical changes) formed the non-labouring group $(\mathrm{n}=3)$. Women who entered spontaneous and established labour (at least $2 \mathrm{~h}$ ) but required emergency caesarean section formed the labouring group $(\mathrm{n}=17)$. Clinical indications for emergency caesarean section included breech (1), fetal distress (5), prolonged labour and failure to progress in labour (7) and previous caesarean section (4). A labour is defined as prolonged when its duration exceeds 18 to 24 hours for nulliparous and 14 hours in multiparous.

Similarly we have obtained clinical samples for preterm labour $(n=6)$ and preterm non labour $(\mathrm{n}=5)$. Clinical indications for CS in the preterm non-labour group were preterm premature rupture of membranes (PPROM) and chorioamnionitis, tranverse lie with cord prolapse after PPROM, and unprovoked fetal distress. The non pregnant samples $(\mathrm{n}=18)$ were obtained from premenopausal women undergoing hysterectomy for non malignant conditions. Myometrial tissue samples (both pregnant and non pregnant) were collected from the lower uterine segment, immediately snap frozen in liquid nitrogen and stored at $-80^{\circ} \mathrm{C}$ for subsequent mRNA analysis. Ethical approval has been obtained by the local ethics committee.

\section{Cell culture}

ULTR cells were a kind gift from Dr Xiaolan Cui (Department of Obstetrics and Gynecology, University of Cincinnati, USA). The cells were maintained at standard culture conditions of $5 \% \mathrm{CO}_{2}$ in air at $37^{\circ} \mathrm{C}$. ULTR cells were cultured in DMEM + Glutamax-1 (Fisher, UK) containing 10\% heat-inactivated fetal bovine serum (FBS), and 0.5\% penicillin-streptomycin.

\section{RNA isolation and CDNA synthesis for qRT-PCR}

Total ribonucleic acid was isolated using an RNA extraction kit (Sigma), according to the manufacturer's instructions. RNA concentration was determined by spectrophotometric analysis (NanoDrop; Thermo Scientific). RNA (200ng from placental tissue and $500 \mathrm{ng}$ from cell lysates) was reverse-transcribed into cDNA using 5 IU/ $\mu 1$ Superscript II reverse transcriptase (Invitrogen). 


\section{Quantitative RT-PCR}

Relative expression of the genes of interest was assessed by quantitative RT-PCR (qRTPCR) on an ABI7900 instrument (Applied Biosystems) using SYBR ${ }^{\circledR}$ Green-PCR reaction mixture (Sigma-Aldrich, UK) and the primers as previously described [18]. As a negative control for all the reactions, distilled water was used in place of the cDNA. RNAs were assayed from two to three independent biological replicates. The RNA levels were expressed as a relative quantification (RQ) value, using Delta $\mathrm{Ct}$ method for comparing relative expression results between treatments in qRT-PCR using primers as previously described [18].

\section{Immunofluorescent analysis}

ULTR cells were fixed in 4\% paraformaldehyde for 10 min prior to washes in PBS and incubation with $10 \%$ bovine serum albumin (BSA) for $1 \mathrm{hr}$. Cells were incubated for $1 \mathrm{hr}$ with antibodies against mTOR, DEPTOR, Rictor and Raptor (Cell Signalling, USA) at a 1:200 dilution in 1\% BSA/PBS. Cells were then washed with PBS prior to an incubation with anti-rabbit IgG-fluorescein isothiocyanate (FITC)-conjugated antibody (Santa Cruz Biotechnology, USA) for $1 \mathrm{hr}$. Slides were washed with PBS and mounted in Vectashield ${ }^{\circledR}$ Mounting Medium (Vector labs) containing the dye 4,6-diamido-2-phenylindole (DAPI) to counterstain nuclei. Images were captured using a Plan Apo Neofluor 63X NA 1.25 oil objective (Zeiss) on a Zeiss Axiovert 200M microscope and viewed using AxioVision software.

\section{Nimblegen Microarray}

ULTR cells were treated with $30 \mathrm{nM}$ P4 for $24 \mathrm{hrs}$ and RNA was obtained using an RNA extraction kit (Sigma), according to the manufacturer's instructions. The RNA was purified and DNase treated using the RNAeasy (Qiagen) clean up protocol supplied by the manufacturer. cDNA was created from 100 ng RNA using the Transplex complete whole transcriptome amplification kit (Sigma). The cDNA sample was RNase (Sigma) treated and cDNA precipitated using the protocol supplied with the Nimblegen Expression Array (Roche). Samples were labelled with Nimblegen labelling kit using $\mathrm{Cy} 3$ and hybridised to a Nimblegen 12 plex $\times 135 \mathrm{k}$ gene Human transcriptome microarray and washed to according to the supplier's protocol. The Nimblegen array slide was scanned at $3 \mu \mathrm{m}$ on an InnoScan 700 Microarray Scanner for TIFF images using Mapix v. 5.1 software. The TIFF images were aligned to their Nimblegen design files and converted into probe intensity values using the Nimblegen DEVA software. The Data was normalised across the array using RMA methods in DEVA. This was imported into DNAstar (ArrayStar Inc.). Pathway analysis was carried out by inputting mTOR-elated genes with $\pm 20 \%$ changes into DAVID v. 6.7 (http:// david.abcc.ncifcrf.gov).

\section{Statistical analysis}

Statistical analysis of the $\Delta \mathrm{Ct}$ values was performed by one-way analysis of variance (ANOVA), followed by Bonferroni Multiple Comparison as a post hoc test using GraphPad Prism 5.0.

\section{Results}

\section{Expression of mTOR, DEPTOR, Rictor, Raptor in human myometrium}

qRT-PCR analyses demonstrated that mTOR was only significantly down-regulated in preterm-labour compared to non pregnant status (Figure 1A). DEPTOR is significantly down-regulated in all the different categories of pregnant myometria when compared to non pregnant myometrium (Figure 1B). In the pregnant myometrium, DEPTOR was up- 
regulated at term non-labour and down-regulated in preterm labour when compared to termlabour (Figure 1B). Rictor showed the least differences in gene expression and there were no apparent differences in its expression across all different groups (Figure 1C). Raptor was significantly up-regulated at term non-labour and down-regulated at preterm labour compared to the non-pregnant group (Figure 1D).

\section{Effects of inflammatory cytokines on mTOR, DEPTOR, Rictor, Raptor in ULTR cells}

Following the mapping of these components in clinical samples, we evaluated the ULTR human myometrial cell line as an in vitro model to further study mTOR signalling. RT-PCR analyses confirmed that all key components are expressed at the mRNA level (Figure 2A). Immunofluorescent analysis was then performed to elucidate protein expression and cellular distribution of these genes. All components appeared to have a strong cytoplasmic distribution with mTOR and Raptor also demonstrating some nuclear staining (Figure 2B).

ULTRs were treated for 24 hrs with IL-1 $\beta$, IL-6, IL-8 and TNF-a ( $1 \mathrm{ng} / \mathrm{mL})$ individually and together as a cytokine mix in attempt to mimic an inflammatory milieu in vitro. Most of the cytokines did not alter gene expression (Fig 3). Treatment with TNF-a significantly downregulated DEPTOR but not mTOR, Raptor or Rictor when compared to basal (unstimulated) levels (Figure 3B). Similarly, incubation of myometrial cells with a mix of all the cytokines did not induce any significant changes in the expression of these genes when compared to control (Figure $3 \mathrm{~A}-\mathrm{D}$ ).

\section{Effects of progesterone (P4) on mTOR signalling components: validation of microarray data}

We performed a gene expression analysis using the NimbleGen chip array in ULTRs treated with 30nM P4 for $24 \mathrm{hrs}$. By analyzing three pairs of independent replicates of P4 treated against unstimulated controls, 1644 significantly differentially expressed genes were identified. We concentrated on the potential effects $\mathrm{P} 4$ can exert on the mTOR pathway (Figure 4). The following genes had lower transcript abundance compared to basal levels: DEPTOR (72\%), GßL (30-50\%), Rictor (29\%), Raptor (18\%), Akt1 (37-60\%), RHEB (17\%), TSC2 (38\%), PRAS40 (17-53\%), IRS1 (40\%), REDD1 (18\%), LKB1 (22\%), STRAD (45\%), MO25 (27\%), HIF1a (21-29\%), VEGF (14-39\%), and AMPK (18\%). Of those only G $\beta$ L, Rictor, and TSC2 appeared to be downregulated significantly (p $\unlhd$.05). On the contrary upregulation following P4 treatment of PKCa (50\%), PDK1 (52-63\%), TSC1 (25-90\%), S6K1 (4-23\%), ERK 1 (66\%), and $\mathrm{ERK}_{2}(22-71 \%)$ was observed.

The qRT-PCR results for the genes in the mTOR pathway (mTOR, DEPTOR, Rictor, Raptor) in an independent validation set were consistent with the results from the microarray data analysis. Treatment of cells with $\mathrm{P} 4(30 \mathrm{nM})$ caused significant down-regulation of mTOR (65\%), DEPTOR (50\%), Rictor (57\%), and Raptor (70\%) when compared with basal levels (Figure 3E).

\section{Discussion}

This study provides the first evidence for differential expression of key signalling components of the mTOR pathway in the human myometrium during pregnancy. In addition, this study is the first to identify and validate the patterns of gene expression associated with myometrial mTOR signalling in P4-treated cells.

Immunofluorescent analysis revealed strong cytoplasmic staining for DEPTOR and Rictor, whereas mTOR and Raptor had cytoplasmic as well as nuclear distribution. These data corroborate our previous findings where a cytosolic expression of mTOR in BeWo and 
JEG-3 cytotrophoblast cell lines [9] and in two ovarian cancer cell lines (SKOV-3 and PEO1) [18] was noted. Other studies have also shown cytoplasmic localisation of mTOR [7, 19], although it has been argued that mTOR can also shuttle between the nucleus and cytoplasm, an event necessary for the maximal activation of S6K1 [20]. It has been proposed that mTORC1 complex is predominantly cytoplasmic whereas the mTORC2 complex is abundant in both compartments [21].

One of the main findings is the significant down-regulation of DEPTOR in all pregnant states when compared to non pregnant controls. DEPTOR binds to both mTORC1 and mTORC2 and knocking down DEPTOR leads to activation of signalling through both complexes [22]. Therefore, we also represented the qRT-PCR data as a ratio of mTOR/ DEPTOR. The ratio of mTOR to DEPTOR was decreased in non-labour irrespective of whether the myometrium was at term (from 2.8 to 1.6 ) or preterm when compared with the labouring samples (from 3.8 to 2.3). Indeed mTORC1 has been shown to be involved with control of cellular proliferation in numerous in vitro as well as in vivo models. For example, there is an increase in the phosphorylation of mTOR and S6K1 during the proliferative phase in the rat myometrium, and treatment of myometrial cells with rapamycin significantly reduced cell proliferation [11]. Our studies tend to corroborate the clinical significance, since the dramatic down-regulation during pregnancy of DEPTOR would allow changes of the myometrial phenotype as activation of the mTORC1 complex can allow progression of myometrium from the proliferative to the synthetic stage.

It has been shown that there is significant and widespread thinning of the myometrium during active labour and that descent of the fetal head during the second stage of labour is associated with a significant relative thickening of the anterior and fundal myometrium [23]. The authors of the study proposed a model where "the myometrium grows symmetrically during pregnancy and thins significantly at all sites during active labour including the fundus". Moreover, it has been proposed that the myometrium might also harbour stem/ progenitor cells that can play a key role in uterine remodelling [24-25]. Of interest, mTOR can regulate smooth muscle differentiation from progenitor cells [26]. During pregnancy, the wet weight of the uterus increases 10 -fold largely due to myometrial smooth muscle cell hypertrophy and hyperplasia. It is attractive to speculate that the down-regulation of the mTOR/DEPTOR ratio in all non-labouring myometrial might compromise the activity of mTORC1 complex thus preventing a full reconstruction of the uterine cavity during pregnancy that can affect the contractile phenotype towards the end. Interestingly, in a study of twin pregnancies, in the absence of uterine contractions or symptoms of preterm labour, twins that delivered preterm had a significantly thinner lower uterine segment at an earlier gestation, compared with twins that delivered at term; suggesting that lower uterine segment thinning occurred earlier in these cases [27].

There is increasing evidence that mTOR signalling is under the influence of steroids. In the myometrium of ovariectomized rats, E2 activated the mTOR signalling pathway [11], whereas we have shown that cortisol-treatment of cytotrophoblast cells down-regulated DEPTOR [10]. Moreover, P4 suppressed mTOR pathway in induced regulatory T cells [iTreg, 28]. iTreg cells were prepared by 5 -day activation of naive $\mathrm{T}$ cells with concanavalin $\mathrm{A}$ and interleukin-2 [28]. However, E2+P4 induced mammary tumour growth in rats via activation of mTOR signalling and phosphorylation of S6K and 4EBP1 [29]. In ovariectomized mice, P4 treatment significantly increased levels of S6K in the dorsal hippocampus [30]. In an attempt to dissect further the effects of P4 on ULTR cells, we performed an mTOR-related gene expression analysis using NimbleGen microarray. We noted down-regulation of a number of transcripts within the mTORC1 and mTORC2 complexes, as well as upstream and downstream effectors in P4 treated samples. We have confirmed these findings using qRT-PCR. Our study follows previous microarray-based 
work, where it was shown that mTOR is one of the most highly up-regulated pathways in leiomyomata [31].

Taken together, the results suggest that in addition to other pleiotropic effects of $\mathrm{P} 4$ at the uterine level, this sex steroid also modulates mTOR signalling which has potential clinical significance. We therefore propose the following model: Early in pregnancy when the P4 levels are low mTOR signalling components are unaffected. As a result, an intact mTOR signalling system favours a state of myometrial hyperplasia. Later on, the rise in P4 levels will antagonize the proliferative effects of E2 and downregulate mTOR signalling. This promotes a state of hypertrophy where there is an increase in the cell size but not in the cell number. At the end of pregnancy "functional" progesterone withdrawal will shift the myometrium from a quiescent state to a contractile one initiating labour.

One of the limitations of this study is that due to ethical restrictions, we could only obtain samples from the lower segment and not from the fundus. However, it has been clearly demonstrated that the increased expression of $I L-8, P G H S-2, I L-1 \beta$ and $C X-43$, i.e. genes associated with labour is greatest in the lower segment [32]. Collectively, the changes in these key genes occurring in the lower uterine segment appear to drive the overall process during labour and subsequently inducing the fundal contractile activity [32].

Future studies should determine the cellular localisation of mTORC1 and mTORC2 complexes in human myometria, especially in tissues from complicated pregnancies such as preterm labour, in order to define whether mTOR shuttling might affect subsequent signalling in certain pathologies. Moreover, future work should elucidate whether P4 can also affect the phosphorylation status of mTOR, S6K, 4EBP, Akt, PKCa and whether it can also affect trafficking of mTOR in myometrial cells.

\section{Acknowledgments}

This research was funded by National Institutes of Health Grant ESO12961. EK and PT should be considered joined last co-authors.

\section{References}

1. Brown EJ, Albers MW, Shin TB, Ichikawa K, Keith CT, Lane WS, Schreiber SL. A mammalian protein targeted by G1-arresting rapamycin-receptor complex. Nature. 1994; 369:756-758. [PubMed: 8008069]

2. Dennis PB, Jaeschke A, Saitoh M, Fowler B, Kozma SC, Thomas P. Mammalian TOR: a homeostatic ATP sensor. Science. 2001; 294:1102-1105. [PubMed: 11691993]

3. Hong-Brown LQ, Kazi AA, Lang CH. Mechanisms mediating the effects of alcohol and HIV antiretroviral agents on mTORC1, mTORC2 and protein synthesis in myocytes. World J Biol Chem. 20120; 3:110-120. [PubMed: 22905289]

4. Jacinto E, Loewith R, Schmidt A, Lin S, Rüegg MA, Hall A, Hall MN. Mammalian TOR complex 2 controls the actin cytoskeleton and is rapamycin insensitive. Nat Cell Biol. 2004; 6:1122-1128. [PubMed: 15467718]

5. Roos S, Powell TL, Jansson T. Placental mTOR links maternal nutrient availability to fetal growth. Biochem Soc Trans. 2009; 37:295-298. [PubMed: 19143650]

6. Wen HY, Abbasi S, Kellems RE, Xia Y. mTOR: a placental growth signalling sensor. Placenta. 2005; 26:S63-69. [PubMed: 15837070]

7. Roos S, Jansson N, Palmberg I, Säljö K, Powell TL, Jansson T. Mammalian target of rapamycin in the human placenta regulates leucine transport and is down-regulated in restricted fetal growth. J Physiol. 2007; 582:449-459. [PubMed: 17463046] 
8. Busch S, Renaud SJ, Schleussner E, Graham CH, Markert UR. mTOR mediates human trophoblast invasion through regulation of matrix-remodeling enzymes and is associated with serine phosphorylation of STAT3. Exp Cell Res. 2009; 315:1724-1733. [PubMed: 19331815]

9. Mparmpakas D, Zachariades E, Foster H, Kara A, Harvey A, Goumenou A, Karteris E. Expression of mTOR and downstream signalling components in the JEG-3 and BeWo human placental choriocarcinoma cell lines. Int J Mol Med. 2010; 25:65-69. [PubMed: 19956903]

10. Mparmpakas D, Zachariades E, Goumenou A, Gidron Y, Karteris E. Placental DEPTOR as a stress sensor during pregnancy. Clin Sci (Lond). 2012; 122:349-359. [PubMed: 21992080]

11. Jaffer S, Shynlova O, Lye S. Mammalian target of rapamycin is activated in association with myometrial proliferation during pregnancy. Endocrinology. 2009; 150:4672-4680. [PubMed: 19589861]

12. Shynlova O, Tsui P, Jaffer S, Lye SJ. Integration of endocrine and mechanical signals in the regulation of myometrial functions during pregnancy and labour. Eur J Obstet Gynecol Reprod Biol. 2009; 144:S2-10. [PubMed: 19299064]

13. Abitbol CL, Rodriguez MM. The long-term renal and cardiovascular consequences of prematurity. Nat Rev Nephrol. 2012; 8:265-274. [PubMed: 22371245]

14. Agrawal V, Hirsch E. Intrauterine infection and preterm labor. Semin Fetal Neonatal Med. 2012; 17:12-19. [PubMed: 21944863]

15. Shynlova O, Lee YH, Srikhajon K, Lye S. Physiologic Uterine Inflammation and Labor Onset: Integration of Endocrine and Mechanical Signals. Reprod Sci. 2013; 20:154-167. [PubMed: 22614625]

16. Hardy DB, Janowski BA, Corey DR, Mendelson CR. Progesterone receptor plays a major antiinflammatory role in human myometrial cells by antagonism of nuclear factor-kappaB activation of cyclooxygenase 2 expression. Mol Endocrinol. 2006; 20:2724-2733. [PubMed: 16772530]

17. Zachariades E, Mparmpakas D, Thomas P, Rand-Weaver M, Karteris E. Crucial cross-talk of interleukin-1 $\beta$ and progesterone in human choriocarcinoma. Int J Oncol. 2012; 40:1358-1364. [PubMed: 22294087]

18. Foster H, Coley HM, Goumenou A, Pados G, Harvey A, Karteris E. Differential expression of mTOR signalling components in drug resistance in ovarian cancer. Anticancer Res. 2010; 30:3529-3534. [PubMed: 20944133]

19. Liu Y, Hidayat S, Su WH, Deng X, Yu DH, Yu BZ. Expression and activity of mTOR and its substrates in different cell cycle phases and in oral squamous cell carcinomas of different malignant grade. Cell Biochem Funct. 2007; 25:45-53. [PubMed: 16927414]

20. Bachmann RA, Kim JH, Wu AL, Park IH, Chen J. A nuclear transport signal in mammalian target of rapamycin is critical for its cytoplasmic signaling to S6 kinase 1. J Biol Chem. 2006; 281:73577363. [PubMed: 16407298]

21. Rosner M, Hengstschläger M. Detection of cytoplasmic and nuclear functions of mTOR by fractionation. Methods Mol Biol. 2012; 821:105-124. [PubMed: 22125063]

22. Peterson TR, Laplante M, Thoreen CC, Sancak Y, Kang SA, Kuehl WM, Gray NS, Sabatini DM. DEPTOR is an mTOR inhibitor frequently overexpressed in multiple myeloma cells and required for their survival. Cell. 2009; 137:873-886. [PubMed: 19446321]

23. Buhimschi CS, Buhimschi IA, Malinow AM, Weiner CP. Myometrial thickness during human labor and immediately post partum. Am J Obstet Gynecol. 2003; 188:553-559. [PubMed: 12592271]

24. Teixeira, J.; Rueda, BR.; Pru, JK. StemBook. Vol. 2008. Cambridge (MA): Harvard Stem Cell Institute; 2008 Sep 30. Uterine stem cells. Available from http://www.ncbi.nlm.nih.gov/books/ NBK27042/

25. Maruyama T, Masuda H, Ono M, Kajitani T, Yoshimura Y. Human uterine stem/progenitor cells: their possible role in uterine physiology and pathology. Reproduction. 2010; 140:11-22. [PubMed: 20457595]

26. Hegner B, Lange M, Kusch A, Essin K, Sezer O, Schulze-Lohoff E, Luft FC, Gollasch M, Dragun D. mTOR regulates vascular smooth muscle cell differentiation from human bone marrow-derived 
mesenchymal progenitors. Arterioscler Thromb Vasc Biol. 2009; 29:232-238. [PubMed: 19074484]

27. Sfakianaki AK, Buhimschi IA, Pettker CM, Magloire LK, Turan OM, Hamar BD, Buhimschi CS. Ultrasonographic evaluation of myometrial thickness in twin pregnancies. Am J Obstet Gynecol. 2008; 198:1-10. [PubMed: 18166295]

28. Lee JH, Lydon LP, Kim CH. Progesterone suppresses the mTOR pathway and promotes generation of induced regulatory T cells with increased stability. Eur J Immunol. 201210.1002/eji.201142317

29. Arumugam A, Parada J, Rajkumar L. Mammary cancer promotion by ovarian hormones involves IGFR/AKT/mTOR signaling. Steroids. 2012; 77:791-797. [PubMed: 22465879]

30. Orr PT, Rubin AJ, Fan L, Kent BA, Frick KM. The progesterone-induced enhancement of object recognition memory consolidation involves activation of the extracellular signal-regulated kinase (ERK) and mammalian target of rapamycin (mTOR) pathways in the dorsal hippocampus. Horm Behav. 2012; 61:487-495. [PubMed: 22265866]

31. Crabtree JS, Jelinsky SA, Harris HA, Choe SE, Cotreau MM, Kimberland ML, Wilson E, Saraf KA, Liu W, McCampbell AS, Dave B, Broaddus RR, BrownL EL, Kao W, Skotnicki JS, AbouGharbia M, Winneker RC, Walker CL. Comparison of human and rat uterine leiomyomata: identification of a dysregulated mammalian target of rapamycin pathway. Cancer Res. 2009; 69:6171-6178. [PubMed: 19622772]

32. Tattersall M, Engineer N, Khanjani S, Sooranna SR, Roberts VH, Grigsby PL, Liang Z, Myatt L, Johnson MR. Pro-labour myometrial gene expression: are preterm labour and term labour the same? Reproduction. 2008; 135:569-579. [PubMed: 18367515] 


\section{highlights}

- DEPTOR was in all pregnant states when compared to non-pregnant myometria.

- The human myometrial ULTR cell line express mTORC1 and mTORC2 components.

- Progesterone down-regulated mTOR, DEPTOR, Rictor and Raptor in myometrial cells.

- Microarray revealed down-regulation of mTOR-related signalling components in P4-treated samples. 
A.

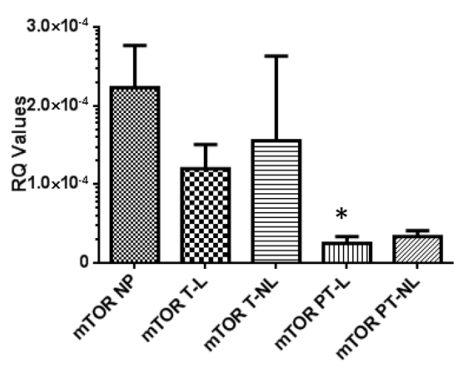

C.

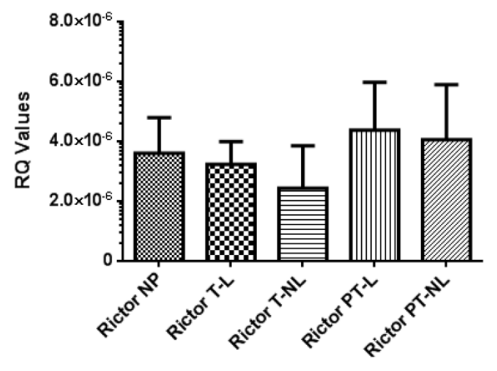

B.

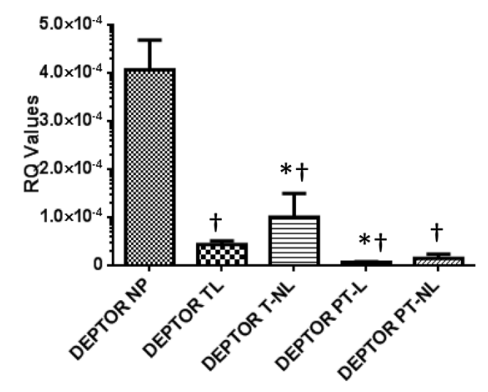

D.

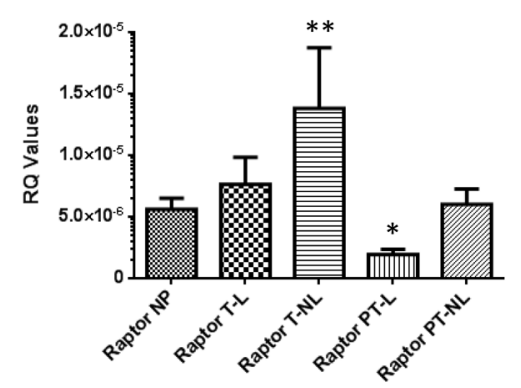

Figure 1. Differential regulation of mTOR-related components in human myometria: downregulation of DEPTOR in all pregnant states when compared to non-pregnant controls Quantitative analyses of mTOR (A), DEPTOR (B), Rictor (C), and Raptor (D) mRNA in human myometria. NP: non pregnant, TL: term labour, T-NL: term no labour, PT-L: preterm labour, PT-NL: preterm no labour. * $\mathrm{p}<0.05$ compared to NP levels for mTOR and Raptor, $\dagger \mathrm{p}<0.05$ compared to NP for DEPTOR and * $\mathrm{p}<0.05$ for DEPTOR compared to TL samples. 
A.

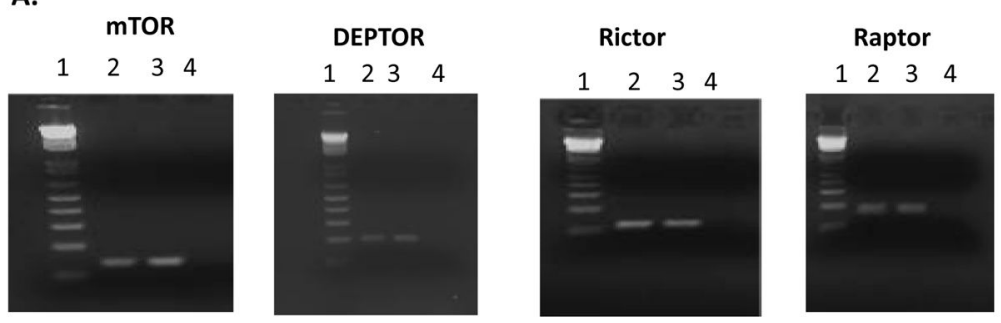

B.
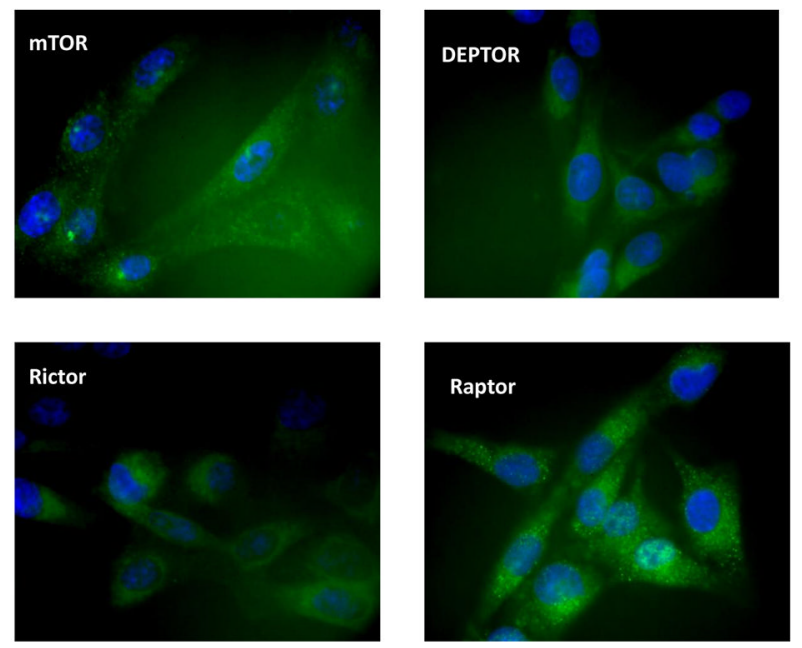

Figure 2. The ULTR myometrial cell line expresses mTOR, DEPTOR, Rictor and Raptor at mRNA and protein level

Immunofluorescent analysis indicates a strong cytoplasmic distribution with mTOR and Raptor. Expression and cellular distribution of mTOR components in ULTR cells. Panel A: Lane 1 DNA marker, Lane 2 cDNA from BeWo cells (positive control), lane 3: cDNA from ULTR cells, and lane 4: negative control RT-). Panel B: Representative images showing cellular distribution of mTOR, DEPTOR, Rictor and Raptor in ULTR cells using immunofluorescent analysis. Magnification x40 
A.

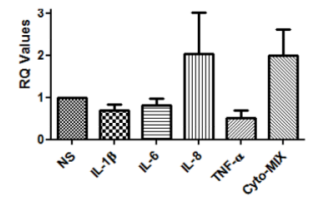

B.

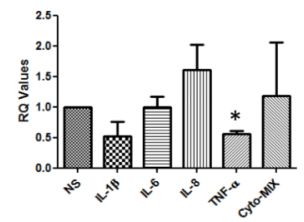

c.

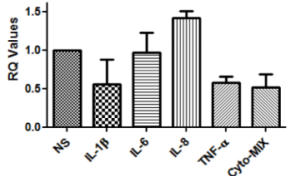

D.

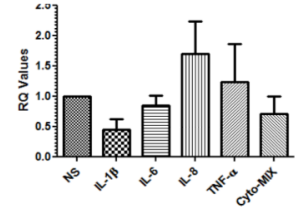

E.

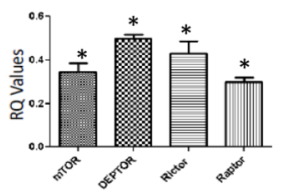

Figure 3. Progesterone but not IL-1 $\beta$, IL-6, IL-8 down-regulated mTOR, DEPTOR, Rictor and Raptor, compared to unstimulated control cells

Myometrial cells were treated with for 24 hrs with IL-1 $\beta$, IL-6, IL-8 and TNF-a $(1 \mathrm{ng} / \mathrm{mL})$ and levels of mTOR (A), DEPTOR (B), Rictor (C) and Raptor (D) were assessed. Only TNF-a significantly $(* \mathrm{p}<0.05)$ downregulated DEPTOR's expression. Panel E: Cells were treated with 30nM P4 for 24 hrs and the levels of mTOR, DEPTOR, Rictor, and Raptor were assessed compared to basal levels that were set up as 1.0 in terms of RQ value $(* p<0.05$ compared to basal levels). 


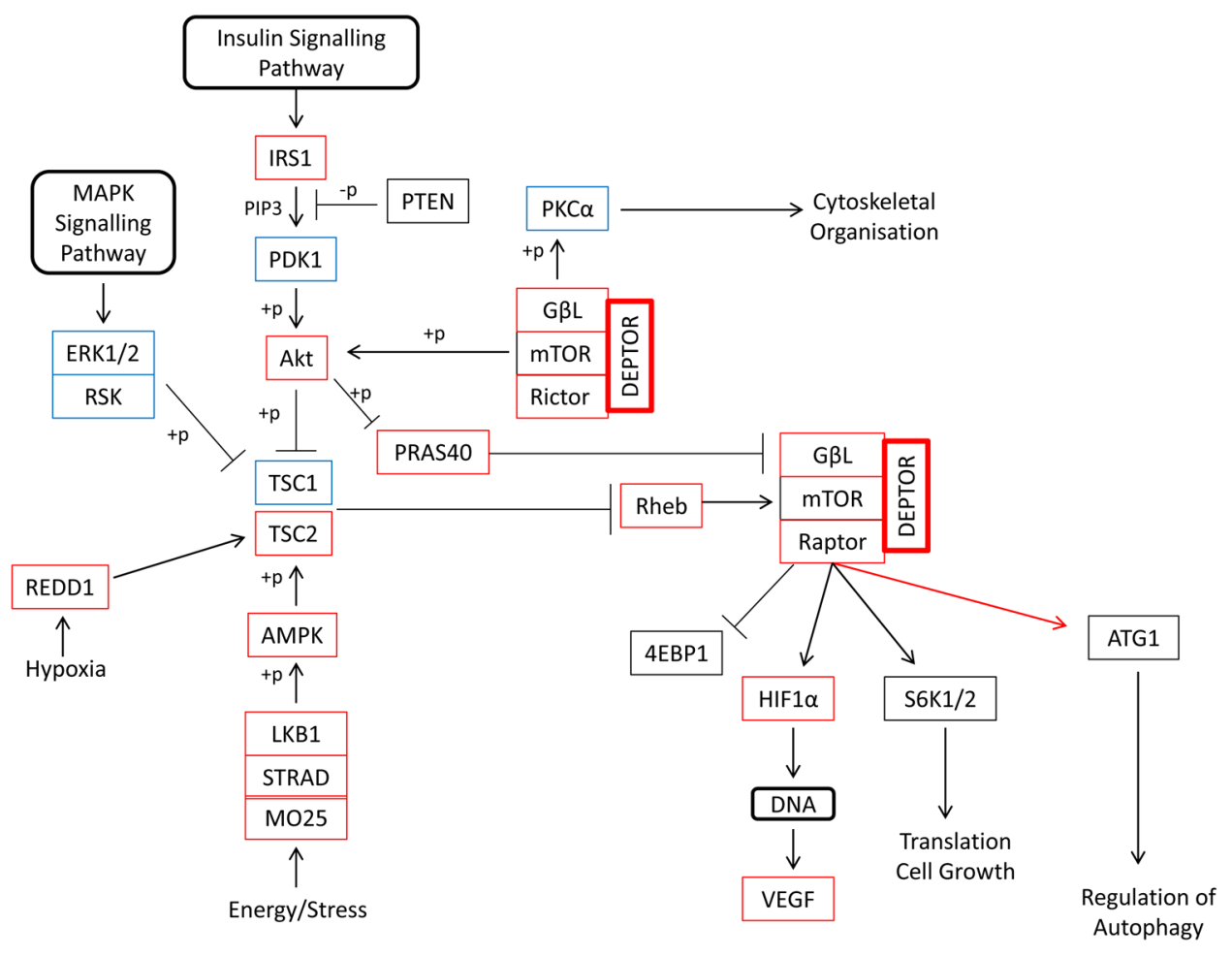

Figure 4. NimbleGen microarray revealed down-regulation of a number of mTOR-related transcripts, as well as upstream and downstream effectors in P4-treated samples

Functional annotation table of the components related to the mTOR pathway that have been downregulated (red rectangle) and upregulated (blue rectangle) following P4 treatment based on Nimblegen array data. 